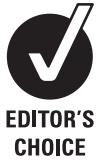

${ }^{1}$ Department of Internal Medicine, Oldenburg Municipal Hospital, Oldenburg, Germany;

${ }^{2}$ Central Interdisciplinary Endoscopy Unit, Department of Gastroenterology, Charité Medical University Hospitals Virchow Campus, Berlin;

${ }^{3}$ Department of Internal Medicine I, Munich-Neuperlach Municipal Hospital, Munich, Germany; ${ }^{4}$ Department of Internal Medicine II, Chemnitz Hospital, Chemnitz, Germany:

${ }^{5}$ Department of Internal

Medicine III, Wald-Krankenhaus Gera, Gera, Germany:

${ }^{6}$ Department of Internal

Medicine II, Technical University of Munich, Munich, Germany;

${ }^{7}$ Department of Internal

Medicine, Siloah Hospital,

Hannover, Germany

Correspondence to:

Professor T Rösch, Klinik und

Poliklinik für Interdisziplinäre

Endoskopie, Martinistr 52,

20246 Hamburg, Germany;

t.roesch@uke.de

Revised 16 February 2009

Accepted 17 February 2009

Published Online First

28 April 2009

\title{
Transluminal endoscopic necrosectomy after acute pancreatitis: a multicentre study with long-term follow-up (the GEPARD Study)
}

\author{
H Seifert, ${ }^{1}$ M Biermer, ${ }^{2}$ W Schmitt, ${ }^{3}$ C Jürgensen, ${ }^{4}$ U Will, ${ }^{5}$ R Gerlach, ${ }^{5}$ C Kreitmair, \\ A Meining, ${ }^{6}$ T Wehrmann, ${ }^{7}$ T Rösch ${ }^{2}$
}

\section{ABSTRACT}

Background: As with endoscopic transmural drainage of peripancreatic fluid collections, the same transluminal access can be expanded to introduce an endoscope through the gastrointestinal wall into the retroperitoneum and remove infected pancreatic necroses under direct visual control. This study reports the first large series with long-term follow-up.

Methods: Data for all patients undergoing transluminal endoscopic removal of (peri)pancreatic necroses between 1999 and 2005 in six different centres were collected retrospectively, and the patients were followed up prospectively until 2008. The initial patient and treatment outcome data were recorded, as were long-term results. Results: Ninety-three patients (63 men, 30 women; mean age 57 years) underwent a mean of six interventions starting at a mean of 43 days after an attack of severe acute pancreatitis. After establishment of transluminal access to the necrotic cavity and subsequent endoscopic necrosectomy, initial clinical success was obtained in $80 \%$ of the patients, with a $26 \%$ complication and a $7.5 \%$ mortality rate at 30 days. After a mean follow-up period of 43 months, $84 \%$ of the initially successfully treated patients had sustained clinical improvement, with $10 \%$ receiving further endoscopic and $4 \%$ receiving surgical treatment for recurrent cavities; $16 \%$ suffered recurrent pancreatitis.

Conclusions: Direct transluminal endoscopic removal of pancreatic necroses is associated with good long-term maintenance of the high initial efficacy; complications can occur, with an associated mortality of around $7.5 \%$. Further studies are necessary in order to optimise endotherapy and define its role in relation to surgery in the clinical management of such patients.

Endoscopic transluminal access has recently been attracting strong interest as a way of obtaining access to the abdominal cavity without the need to create scars. This so-called "natural orifice transluminal endoscopic surgery" (NOTES) approach is currently being evaluated experimentally for a variety of intra-abdominal procedures. ${ }^{1}$ Transluminal retroperitoneal endoscopy for the debridement of solid infected necroses in patients with severe necrotising pancreatitis is the first clinical application of transluminal interventions, and was pioneered a few years ago. ${ }^{2}$ It followed endoscopic transmural stent drainage of pseudocysts and abscesses, replacing the respective surgical techniques. ${ }^{3-5}$ Endoscopic ultrasonography (EUS), then newly emerging, allowed transmural puncture and drainage even in more complex anatomical conditions; eg, without bulging of the cyst into the gastrointestinal wall. ${ }^{6}$ However, without direct visual control through endoscopy, this type of intervention was only possible in limited or partially liquefied necrotic lesions. ${ }^{7}$ The next step was to actively work outside the gastrointestinal tract with an endoscope, by expanding the transgastric access to more extended necroses. Widening the transmural fenestrations made it possible to carry out retroperitoneal endoscopic interventions. This report describes the first long-term results of a large multicentre series on this new minimally invasive approach.

\section{PATIENTS AND METHODS \\ Patient inclusion}

All patients treated in six different tertiary referral centres between 1999 and 2005 were included in this retrospective analysis, with variable starting points in each centre (depending on the various time points at which the new technique was established locally), but with a common endpoint in 2005. A prospective follow-up protocol of patients started in 2004 and ended in 2008. The cohort includes three patients whose initial procedural results were subjects of the first publication of this new endoscopic approach ${ }^{2}$ which did not include follow-up data reported here.

Patients in whom direct endoscopic therapy was started after transluminal access to the collection were included. The indication for the endoscopic transmural approach was an infected necrosis in patients suffering from constant or intermittent fever, increasing laboratory parameters suggesting infection with worsening tendency or not subsiding by conservative means (leucocytes, C-reactive protein) and in a substantially reduced clinical condition 2-6 weeks after an acute attack of pancreatitis. Conservative measures as well as drainage methods alone, including irrigation by nasocystic catheters, had failed to resolve this situation. The initial patient assessment included the clinical picture, laboratory results, and imaging procedures such as ultrasound and computed tomography (CT). Collections containing significant amounts of solid necroses (judged as $>50 \%$ of echogenic material by ultrasonography/endoscopic ultrasonography (US/EUS)) were included. In contrast, collections which mainly consisted of fluid, abscesses with little necrotic material and those collections/necroses which extended into the lower abdomen/pelvis were excluded. Endoscopic retrograde cholangiopancreatography (ERCP) was 
Table 1 Clinical details of the study patients

\begin{tabular}{|c|c|}
\hline & No (\%) \\
\hline Patients & 93 \\
\hline Sex (M:F) & $63: 30$ \\
\hline Age (years; mean/range) & $57(21-82)$ \\
\hline \multicolumn{2}{|l|}{ Aetiology of pancreatitis } \\
\hline Alcoholic & $28(30)$ \\
\hline Biliary & $43(46)$ \\
\hline Post-ERCP* & $5(5)$ \\
\hline Other & $17(18)$ \\
\hline \multicolumn{2}{|l|}{ Predominant signs and symptoms } \\
\hline Sepsis: & $66(71)$ \\
\hline Abdominal pain & $82(88)$ \\
\hline Gastric obstruction/vomiting & $65(70)$ \\
\hline Bile duct compression & $19(20)$ \\
\hline Splenoportal thrombosis & $33(36)$ \\
\hline Pancreatic ascites & $39(42)$ \\
\hline Pleural effusion & $24(26)$ \\
\hline $\begin{array}{l}\text { Spontaneous perforation into } \\
\text { gastrointestinal tract }\end{array}$ & $10(11)$ \\
\hline \multicolumn{2}{|l|}{$\begin{array}{l}\text { Delay from onset of symptoms to } \\
\text { first necrosectomy }\end{array}$} \\
\hline$<10$ days & $2(2)$ \\
\hline 10-20 days & $21(23)$ \\
\hline $21-40$ days & $33(36)$ \\
\hline $41-60$ days & $18(19)$ \\
\hline$>61$ days & $19(20)$ \\
\hline \multicolumn{2}{|l|}{ Main location of cavity } \\
\hline Head and head/body & 21 \\
\hline Body and tail & 42 \\
\hline Entire pancreas & 30 \\
\hline Cavity size (cm; mean/range) & $11.4(4.0-25.0)$ \\
\hline \multicolumn{2}{|c|}{$\begin{array}{l}\text { *Including one patient with endoscopic papillectomy. } \\
\text { †lncluding two postoperative, one due to panarteriitis nodosa, one } \\
\text { ischaemic and two cases with no other findings but pancreas divisum. } \\
\text { \$As defined by one of the following: fever, C-reactive protein raised } \\
>8 \times \text { upper limit of normal, bacteraemia, positive culture from cavity } \\
\text { biopsy. } \\
\text { ERCP, endoscopic retrograde cholangiopancreatography. }\end{array}$} \\
\hline
\end{tabular}

carried out when indicated (when there was a suspicion of ductal leakage into the fluid collection).

\section{Endotherapy}

Patients underwent endoscopic treatment of their pancreatic necroses, involving the following steps:

- Creation of a transgastric or transduodenal access to the retroperitoneal cavity using endoscopic or endosonographic guidance, followed by insertion of two or more stents and in some cases nasocystic irrigation catheters. EUS or endoscopic guidance was used to gain initial access; patient selection for either technique was determined by the endoscopic aspect; cases with clear and massive bulging including signs of inflammation and incipient perforation were treated by initial endoscopic access, whereas all other cases were initially drained under endosonographic guidance. For stenting, multiple stents of variable form and diameter were used, sometimes with a nasocystic irrigation and suction catheter, to keep the access open.

- In the next session, balloon dilation (maximal diameter 15 or $20 \mathrm{~mm}$ ) was carried out to permit the introduction of a conventional gastroscope to allow forceful irrigation and suction, as well as active endoscopic removal of debris using snares, forceps, and stone removal baskets.

- Repeated sessions at intervals of 1-4 days were carried out until all debris and necrotic material had been removed and the walls of the collections could be seen as vital structures. The duration of a session was determined mainly by patient tolerance of sedation or general anaesthesia, but was usually limited to $90 \mathrm{~min}$.

- As the final step, stent drainage of the emptied cavity was carried out for 6-12 weeks and checked using external imaging (ultrasound or CT).

- Any additional interventional measures (eg, ERCP with transpapillary drainage, percutaneous drainage, surgery) were recorded.

\section{Outcome parameters and follow-up}

The main outcome parameter was the long-term clinical success of endoscopic necrosectomy without the need for further interventions (for definitions, see below). The minimum followup period was 2 years, except for those patients who died.

The secondary outcome parameters were:

- Initial treatment success (within 30 days, or at the time of hospital discharge)

- Long-term quality-of-life assessment

- Initial and long-term complications

- Recurrence of necrosis/cysts necessitating repeat interventions, as well as recurrence of pancreatitis

Initial outcome (within 1 month) was assessed as follows:

- Clinical success: complete if the patient was symptom-free, and no further interventions were required. Failure: failure to resolve the collection, requiring other interventions, and/ or failure to significantly improve the patient's symptoms, and/or complications necessitating a switch to other therapies (mostly surgery), and/or mortality.

- Radiological (morphological) success: complete if there was no cyst/fluid collection; partial if there was some cystic cavity remaining $(<3 \mathrm{~cm})$, not requiring further interventional treatment. Failure: collection unchanged.

- Complications and mortality

Follow-up was started at the time of hospital discharge, and data were collected by direct or telephone interviews with patients, relatives, and/or family physicians in a standardised

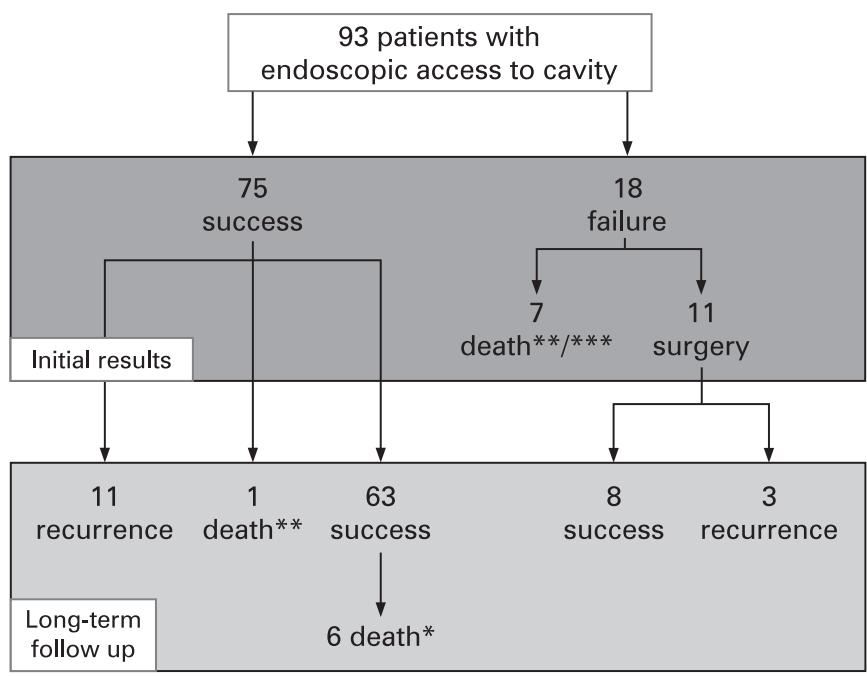

* fatalties not related to pancreatitis or necrosectomy were regarded as succes

** these fatalties were directly related to pancreatitis, necrosectomy or recurrent pancreatitis

*** including one patient who died within the initial hospitalization after surgery for treatment failure

Figure 1 Flow-chart showing the treatment course for the study patients, initially and long-term (for definitions, see text). 
fashion by a research fellow supervised by one gastroenterologist at each respective centre. The follow-up consisted of assessment of the clinical situation, relevant laboratory parameters, and follow-up imaging procedures to the extent that they were available at different time points (US, EUS, CT). Further specific interventions and pancreatitis-related hospital admissions were also recorded. Late evaluation (long-term outcome) of patients with initial endotherapy success was performed up to 2008 on the basis of the following definitions:

- Clinical well-being with no or minimal fluid collection $(<3 \mathrm{~cm}$, no infection), with no or minimal symptoms, and no need for further intervention (yes/no). Pain attributed to the underlying pancreatitis, without morphological evidence of a residual or recurrent cavity, was not considered. Recurrence of a cystic lesion, abscess or necrosis in the same location, with clinical symptoms of varying severity, and/or the need for surgical therapy were regarded as failure.

- Recurrence of underlying pancreatitis, without local complications such as abscess or necrosis, was considered separately.

Mainly descriptive statistics were used.

\section{RESULTS}

\section{Patient data}

Ninety-three patients (68\% male, mean age 57 years (range, 21-82) were included in the six centres (table 1), 48 of whom were referred from elsewhere to these centres before direct endoscopic therapy was started after transluminal access to the collection. All of the patients were significantly ill, with four patients being transferred from other hospitals in septic shock, 19 patients

Table 2 Long-term outcome in 86 surviving patients

\begin{tabular}{|c|c|c|}
\hline & $\begin{array}{l}\text { Patients with } \\
\text { initially successful } \\
\text { endotherapy }(87 \%) \\
(n=75)\end{array}$ & $\begin{array}{l}\text { Patients with initial } \\
\text { surgery (failure of } \\
\text { endotherapy) }(13 \%) \\
(n=11)\end{array}$ \\
\hline & No (\%) & No (\%) \\
\hline \multicolumn{3}{|l|}{ Overall long-term success } \\
\hline Clinical success & $63(84)$ & $8(73)$ \\
\hline Clinical failure & $12(16)$ & $3(27)$ \\
\hline \multicolumn{3}{|l|}{ Clinical characteristics } \\
\hline Recurrent pancreatic pain & $15(20)$ & $4(36)$ \\
\hline Regular use of analgetics & $10(13)$ & $4(36)$ \\
\hline Enzyme therapy & $23(31)$ & $8(73)$ \\
\hline Pre-existing diabetes & $24(32)$ & $4(36)$ \\
\hline $\begin{array}{l}\text { Newly developed } \\
\text { diabetes }\end{array}$ & $15(20)$ & $3(27)$ \\
\hline Insulin dependence & $26(35)$ & $4(36)$ \\
\hline \multicolumn{3}{|l|}{ Morphology of cavity } \\
\hline Remaining cavity/cyst & $9(12)^{*}$ & 0 \\
\hline Recurrence of cavity/cyst & $8(11)$ & $4(36)$ \\
\hline \multicolumn{3}{|l|}{ Further treatment } \\
\hline Re-hospitalisation total & $15(20)$ & $5(45)$ \\
\hline Endoscopic cyst drainage & 7 (9) & $3(27)$ \\
\hline Repeat endotherapy & $2(3)$ & - \\
\hline $\begin{array}{l}\text { Pancreatic surgery during } \\
\text { follow-up }\end{array}$ & $3(4)$ & - \\
\hline \multicolumn{3}{|l|}{ Mortality } \\
\hline Total & $7(9) \dagger$ & $0(0)$ \\
\hline Related to pancreatitis & $1(1)$ & 0 \\
\hline
\end{tabular}

*Of these nine patients, two underwent endotherapy and seven had long-term spontaneous resolution.

†For the patients who died (all but one due to unrelated reasons), clinical information after endotherapy was available; except for one patient who denied further treatment of necrosis and sepsis none of the patients had any symptoms, and they were all classified as having had clinical success. being treated in the intensive care unit (ICU) directly after hospital admission (20\%). Sixty-six patients $(76 \%)$ were showing signs of generalised sepsis, 82 patients $(88 \%)$ were reporting strong abdominal pain and depended on wide (mostly intravenous) analgesic treatment, and all had been receiving various forms of intravenous antibiotic therapy (usually broadspectrum antibiotics) for more than 1 week. All collections contained significant and variable amounts of solid debris and/ or frankly visible pus. Bacteriological analysis revealed positive cultures in 50 cases; it was positive for various bacteria only in 23 cases and revealed Candida in eight.

Fifty-four patients had previously undergone percutaneous (and/or transgastric) drainage by percutaneous radiological drainages or transgastric implantation of stents; the necrotic collections did not resolve by drainage alone, however, and patients were therefore referred for direct endoscopic necrosectomy; in all cases of percutaneous drainages, the drainage pathway was not considered suitable for direct percuteaneous endoscopic necrosectomy. Three patients had had previous surgical necrosectomy and partial pancreatectomy 133 days, 530 days and 5 years before endoscopic necrosectomy.

\section{Endoscopic procedure}

The time from the onset of acute complicated pancreatitis to necrosectomy was a mean of 41 days (range, 4-158 days); apart from two cases with early intervention who had necrotic pancreatitis on the basis of underlying chronic pancreatitis, the minimum time span, however, was 11 days in the remaining 91 patients, in the period of late and local complications. The time between admission to the respective centre (including referrals) and performance of necrosectomy was a mean of 15 days (range, 0-81 days); indications and delay are detailed in table 1. The duration of hospital stay for necrosectomy in the referral centres was a mean of 46 days (range, 8-170 days), including surgical cases.

For endotherapy, all patients were treated under significant sedation, and EUS was used in most of them to gain access. In detail, patients received intravenous sedation with midazolam 36 $(39 \%)$ (mostly in combination with opioids or ketanest) or propofol $57(61 \%)$. Access to the cavity was created by endoscopic puncture into a clearly bulging lesion as described above $(n=18)$ or in most cases $(n=87)$ with endosonographic guidance $(n=70)$ or, in five further cases, by using a spontaneous perforation of the cyst into the gastrointestinal lumen. Transgastric and transduodenal access was chosen in 80 and 12 cases, respectively. One patient who presented with a large mediastinal abscess extending from the retroperitoneal cavity around the pancreatic tail was successfully treated primarily via a transgastric access which was followed by a transesophageal fenestration. A mean of 6.2 necrosectomy sessions (range, 1-35) were carried out; 18\% of the patients had one session, and the remaining patients had a mean of 7.5 sessions (2-35).

ERCP was performed in 63 patients either before or during the transluminal treatment sessions, and it was followed by ductal endotherapy (sphincterotomy and/or stenting) in 45 cases.

\section{Initial outcome}

An overview is presented in fig 1 .

\section{Clinical and radiological success}

Clinical success (with endoscopic treatment only) was achieved in $81 \%$ of the patients (75 of 93); 48 of these ( $52 \%$ of all 
Table 3 Patients' reported quality of life on long-term follow-up

\begin{tabular}{|c|c|c|c|c|}
\hline \multirow[b]{2}{*}{ Deceased } & \multicolumn{2}{|c|}{$\begin{array}{l}\text { Patients with initial endotherapy } \\
\text { success }(87 \%)(\mathrm{n}=75)\end{array}$} & \multicolumn{2}{|c|}{$\begin{array}{l}\text { Patients with initial surgery (failure of } \\
\text { endotherapy) }(13 \%)(n=11)\end{array}$} \\
\hline & \multicolumn{2}{|l|}{7} & \multicolumn{2}{|l|}{0} \\
\hline \multirow{2}{*}{$\begin{array}{l}\text { Duration of follow-up (in the } \\
\text { remaining patients) }\end{array}$} & \multicolumn{2}{|c|}{ Mean, 50 months; range 15-96 months } & \multicolumn{2}{|c|}{ Mean, 53 months; range $15-93$ months } \\
\hline & $(n=68)$ & Per cent (of 68) & $(n=11)$ & Per cent (of 11) \\
\hline \multicolumn{5}{|l|}{ Working status } \\
\hline Still working & 32 & 47 & 4 & 36 \\
\hline Retired (due to age) & 31 & 46 & 6 & 55 \\
\hline Retired (due to disease) & 5 & 7 & 1 & 9 \\
\hline \multicolumn{5}{|c|}{ Number of patients reporting difficulties with ... } \\
\hline Carrying heavier loads & 19 & 28 & 4 & 36 \\
\hline Walking around the block & 7 & 10 & 3 & 27 \\
\hline Leaving the house & 5 & 7 & 1 & 9 \\
\hline Basic activities & 6 & 9 & 0 & 0 \\
\hline \multicolumn{5}{|c|}{ Number of patients who since necrosectomy had ... } \\
\hline To change their diet & 42 & 62 & 4 & 36 \\
\hline Lost weight & 9 & 13 & 1 & 9 \\
\hline Fever or chills & 4 & 6 & 1 & 9 \\
\hline Jaundice & 2 & 3 & 1 & 9 \\
\hline Felt bloated & 17 & 25 & 4 & 36 \\
\hline \multicolumn{5}{|l|}{ Self-assessment score } \\
\hline \multicolumn{5}{|l|}{0 (very good) to 10 (very poor) } \\
\hline Physical condition & \multicolumn{2}{|c|}{2.47 (range, $0-10$ ) } & \multicolumn{2}{|c|}{3.82 (range, $0-10$ ) } \\
\hline Quality of life & \multicolumn{2}{|c|}{2.35 (range, $0-10$ ) } & \multicolumn{2}{|c|}{3.54 (range, $0-10$ ) } \\
\hline
\end{tabular}

Differences to $100 \%$ are due to rounding to complete numbers.

patients) also showed complete radiological success (defined as no residual necrosis, cyst on the day of discharge). Initial clinical and radiological failure occurred in 18 patients (19\%) and was followed by surgery in 12 (11 long-term survivors). Eight patients underwent surgery electively during the initial hospital stay due to failure of cyst resolution $(n=7)$ or formation of a colonic fistula $(n=1)$. A further four patients underwent emergency surgery due to acute bleeding $(n=2)$ or intraperitoneal cavity perforation $(n=2)$.

\section{Complications}

Necrosectomy complications were encountered in 24 cases $(26 \%$ morbidity) and consisted of:

- Thirteen cases of bleeding (two who underwent surgery; one death before surgery could be performed)

- Five perforations of the necrosis into the abdominal cavity (two treated surgically, two conservatively and one being fatal due to an inoperable status)

- Two cases with fistula formation

- Two cases of air embolism (one patient with fatal pulmonary embolism and one case of cerebral infarction after air embolism via a persistent foramen ovale)

- Two cases of complications at other organs (all undergoing surgery)

Other complications not directly related to necrosectomy were one case each of oesophageal variceal haemorrhage, seizure, and intracerebral haemorrhage. One patient with pneumoperitoneum developed abdominal skin emphysema that could be treated conservatively.

\section{Mortality}

Seven patients died during the initial hospital stay $(7.5 \%)$, one after surgery and six without prior surgery. One death was due to fatal bleeding, one after air embolism, four due to sepsis in general bad condition and one after surgery due to multiple-organ failure. Some of the deaths occurred after 30 days but within the same hospital stay. Four of the patients with fatal outcome were transferred to the centre from other hospitals in septic shock with surgery not being an option due to very poor general condition. These patients were treated endosopically as a last option. Two further patients died during endoscopic necrosectomy with unsuccessful resuscitation before surgery could be performed: one suffered from fatal air embolia, one from a fatal arterial bleeding and one patient died in septic shock due to peritonitis after an unsuccessful surgical attempt of necrosectomy.

\section{Long-term outcome}

The results for the surviving patients (86 patients, see fig 1) are shown in table 2 both for patients who were treated endoscopically $(n=75)$ and surgically $(n=11)$. The follow-up period was a mean of 44 months (range, 4-96), the lower range being due to fatal complications on early follow-up. The median follow-up period among long-term survivors (79 patients) was 50 months (range, 15-96). Two patients were lost for long-term follow-up but could be ultimately contacted 15 and 19 months after necrosectomy; all other surviving patients had a minimal follow-up of 24 months.

During follow-up seven patients died (all in the endoscopic group), accounting for a long-term mortality rate of $7.5 \%$. Of these, however, in six the reasons for their deaths were not directly linked to pancreatitis or necrosectomy. Three patients died due to various carcinomas (two ovarian, and one pancreatic where the pancreatic necrosis of the tail was due to malignant obstruction of the pancreatic duct and chemotherapy could be performed only after endotherapy had removed the infected necrosis) between 6 and 20 months after endotherapy. Two patients died because of other reasons (variceal bleeding due to alcoholic liver cirrhosis, renal tuberculosis with multiple comorbid conditions) 12-49 months after endotherapy. One patient died 20 weeks after hospital discharge due to sepsis 


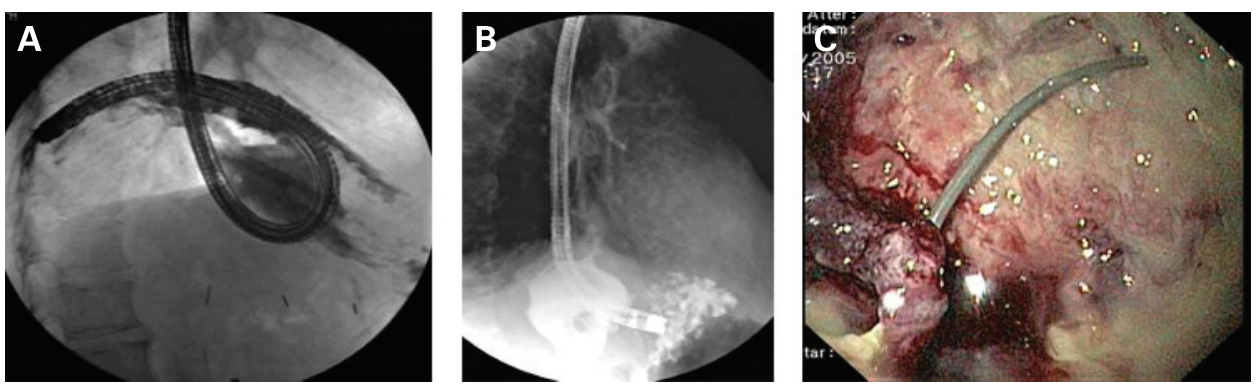

Figure 2 Extended retroperitoneal cavities. (A) The endoscope deeply inserted into the retroperitoneum following the paravertebral necrosis as shown by fluorography. (B) Cavity and fistula to the bronchial system as shown by bronchiography through the endocope positioned inside the retroperitoneal space. (C) The peripheral end of a plastic stent that had been placed in order to drain the pancreatic tail. Most of the necrotic pancreas is now removed leaving the stent as a retroperitoneal transpapillary connection for drainage.

and multiple-organ failure following cholangitis after delayed re-admission, which may have been partly related to the pancreatic damage. The seventh patient died in sepsis due to recurrent necrotising pancreatitis after refusing any further treatment 4 months after discharge from the hospital.

Of the 68 long-term survivors in the group treated endoscopically $(n=75)$, clinical success was maintained in $57(84 \%$ and $61 \%$, respectively, in relation to all 93 patients treated initially). In the 11 cases with secondary clinical failure/recurrence, three patients had recurrent necrosis and underwent surgical necrosectomy (after 13, 27 and 50 months). Six patients with recurrence of larger pseudocysts had to be re-admitted and were again treated endoscopically. Two patients who had been discharged with partial success had to be re-admitted for further sessions of endoscopic necrosectomy. All of these 11 patients were dependent on regular intake of analgesic medication for abdominal pain during the further follow-up. Six further patients nevertheless reported occasional abdominal pain. Of the 11 patients who initially underwent surgery after failed endotherapy and who survived, eight $(73 \%)$ had resolution of the symptoms and cavity. Three patients with recurrent pseudocysts were treated with repeated endoscopic drainage (after 15, 11 and 6 months, respectively). One patient remained symptomatic after initial surgery, and although he did not develop a recurrent cyst or cavity, he underwent surgery for abdominal adhesions after 6 years of follow-up. During follow-up, a further two of the post-surgical patients underwent surgery for incisional abdominal hernias.

Detailed quality-of-life ( $\mathrm{OoL}$ ) data for the long-term survivors are presented in table 3. About $80 \%$ of the endoscopically and surgically treated patients reported a fairly good quality of life. In the endoscopic group, nearly all patients who reported a poor general condition and a reduced QoL index also had reasons other than pancreatitis. In the endotherapy group with longterm follow-up, two patients were suffering from Alzheimer's dementia, one patient from Korsakoff's syndrome, and another is still actively alcohol dependent. One patient has chronic joint problems due to rheumatic arthritis. One patient suffers from severe joint pain due to panarteriitis nodosa. Another patient's quality of life is impaired due to advanced breast cancer.

\section{Summary of results}

An overview of the immediate and long-term results is shown in table 4 and fig 1. An example of endotherapy is shown in figs 2 and 3 .

\section{DISCUSSION}

Transluminal access to the extraluminal retroperitoneal space was initially used by endoscopists to drain pancreatic pseudocysts by inserting transgastric or transduodenal stents; the results (mostly reported in retrospective uncontrolled series) appear to match the success rates with the percutaneous or surgical approach, but with potentially lower complication rates. ${ }^{8}$ This method of endoscopic access requires visualisation of bulging endoluminal cysts. Endoscopic ultrasonography then expanded the indications by providing transluminal access to nonbulging pseudocysts and cavities. ${ }^{6-11}$ Cyst drainage was achieved by inserting one or several stents and a nasocystic irrigation catheter if there was putrid material. With more sticky material, and especially necrotic tissue, drainage alone is often not sufficient. The next major step was therefore to expand the transluminal access to retroperitoneal cavities and use it to introduce an endoscope in order to remove necrotic tissue actively under direct endoscopic vision. ${ }^{7}$ With this type of approach, the primary access is established under endosonographic control, followed by balloon dilation of the orifice, and endotherapy with a conventional or even a largechannel gastroscope involves the use of different endotherapy accessories. Some small and one middle-size series with shortterm follow-up periods have suggested that this form of minimally invasive therapy may be effective and reasonably safe. ${ }^{12-17}$ A recent series of 53 such cases with follow-up of 6 months showed a similar initial success rate and a $21 \%$ rate of minor complications and no mortality. ${ }^{17}$ The differences, especially with respect to complications, may be due to various factors, such as different selection criteria, probably including more severe cases in our series. In the other study, only half of the cases had infected necroses, two of 53 patients were treated on an outpatient basis, and the mean hospital stay or ICU stay are not detailed. ${ }^{17}$ The present study is the first to report the long-term outcome of this new form of endoscopic treatment, and it includes significant numbers of patients treated at several centres. The procedural details of the technique, which was pioneered by Seifert and his group in $2000,{ }^{2}$ are still being further developed and were therefore not fully standardised during the study. This may explain why, initially, and in severely ill patients, the number of sessions was quite high, resembling daily lavage and debridement after surgery. Despite the inherent limitations of a primarily retrospective analysis, the results show that direct endoscopic debridement is feasible, with an initial success rate of $80 \%$ and a $68 \%$ rate of long-term clinical efficacy. Major complications consisted mainly of bleeding and perforation in approximately $26 \%$ of cases, leading to a procedure related mortality rate of $7.5 \%$ in this series. This complication rate might be regarded as acceptable, in view of the much higher rates of complications and mortality reported in surgical series. One of the most feared complications, namely air embolism, was encountered twice during this series and has been reported elsewhere ${ }^{16}$ leading to the 
Figure 3 A patient with a large retroperitoneal necrosis extending into the splenic hilum. (A) Wide gastric fenestration, with solid necrotic masses being extracted from the retroperitoneal space into the gastric lumen.

(B) Endoscopic removal of necrotic material. (C) Endoscopic view of the splenic hilum, which has been cleared of necrotic masses. Minimal residual putrid debris is still visible.
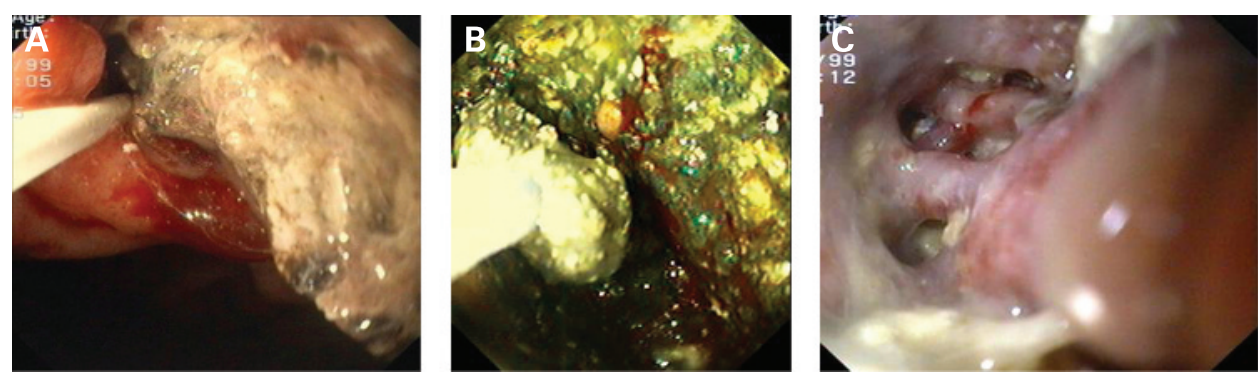

use of carbon dioxide instead of air for endoscopic insufflation in many centres.

The present series suffers from well-known limitations of a retrospective analysis; inclusion criteria were therefore not perfectly uniform, although most centres included patients with later local complications of pancreatic necroses, and in whom fever and signs of infected necrosis predominated. The few early cases were severely ill patients in whom surgery would have been indicated but not deemed possible due to poor general condition. The short delay between onset and intervention in these few cases was taken from the charts, but may be unreliable, so the actual onset of first symptoms before admission may have happened earlier. Patient selection for EUS versus endoscopic guidance was mainly determined by the endoscopic aspect; cases with clear and massive bulging, including signs of inflammation and incipient perforation, were treated by initial endoscopic access, whereas the majority of cases $(70 \%)$ was initially drained under endosonographic guidance. The use of EUS has been recommended for cases without any or without clear cyst bulging into the gastrointestinal lumen, but a recent series suggested that both techniques when appropriately used may lead to a similar clinical outcome. ${ }^{11}$ The choice of stents after initial access as a bridge to the actual endoscopic necrosecomy 1-2 days later was not standardised among centres, although often an additional nasogastric catheter was placed for irrigation and suction. However, in contrast to transluminal cyst drainage, the choice of stents may be of less importance in pancreatic endoscopic necrosectomy, since they mainly serve to keep the access open for subsequent dilatation and endoscope introduction.

From this pilot experience we cannot conclude which endoscopic method is preferable with regards to technical details; we did not perform comparison of different stents, dilatation diameters and delay between initial access and subsequent dilatation and necrosectomy. We believe, however, that the approach commonly used (1-2 days delay, 15-20 mm balloon dilatation) appears to be feasible and that it would not be worthwhile to compare different technical details such as number and diameter of stents. As far as patient selection is concerned, we are convinced that initial necrosectomy attempts in the early phase of pancreatic necrosis are more risky and less successful and should therefore only be considered if all other options are denied. We would also not primarily recommend endotherapy of large areas extending far away from the transluminal access point; ie, those in the pelvis. Although some of the participating centres have meanwhile limited experience with such cases, often percutaneous drainages are necessary in these cases (which can also be used for percutaneous endoscopic necrosectomy). For these reasons we have not included such cases in this retrospective analysis.

The preliminary data presented here need to be compared with that for surgical necrosectomy. The international guidelines propose surgical intervention only in cases of infected necroses and recommend delayed surgery. ${ }^{18}{ }^{19}$ A more recent paper has even suggested that postponing surgery until 30 days or more after the onset of pancreatitis correlates with an improved outcome. ${ }^{20}$ In an analysis of nine studies including a total of 879 patients in the same article, the authors found a positive correlation between timing (delayed) and mortality (decreased). The median mortality rate in this analysis was $25 \%$, with a range of $6-56 \% .^{20} \mathrm{~A}$ recent study specifically looking at early and late complications of surgical necrosectomy, in which half of the procedures were open and half were minimally invasive, reported a $93 \%$ rate of postoperative complications - mostly involving multiple-organ failure $(50 \%)$ or thrombotic/cardiovascular complications (31\%) and a $28 \%$ mortality rate. No differences were observed between the two approaches. ${ }^{21}$ These results were recently confirmed by a small case series on 12 British patients, with morbidity and mortality rates of $67 \%$ and $25 \%$, respectively. ${ }^{22}$ Another study of 59 Dutch patients reported an even higher mortality rate: $47 \%$ in the group undergoing an open abdomen strategy and 33\% in the group with primary closure and continuous postoperative lavage..$^{23}$ Mortality rates of $20-40 \%$ were also confirmed in an analysis of the literature from renowned pancreatic surgery

Table 4 Summary of results

\begin{tabular}{|c|c|c|}
\hline Data & No $(\%)$ & Comments \\
\hline Endoscopic access & 93 & \\
\hline \multicolumn{3}{|l|}{ Initial data } \\
\hline Success & $75(81)$ & \\
\hline Surgery rate & $12(13)$ & $\begin{array}{l}8 \text { due to failure, } 4 \text { due to } \\
\text { complications }\end{array}$ \\
\hline Complications & $24(26)$ & See text \\
\hline Mortality & $7(7.5)$ & $\begin{array}{l}2 \text { during endotherapy, } 5 \text { during } \\
\text { initial hospitalisation }\end{array}$ \\
\hline \multicolumn{3}{|l|}{ Long-term data } \\
\hline Clinical success & $63(68)^{*}$ & \\
\hline Recurrent cavity & $8(9)$ & \\
\hline Recurrent pancreatitis & $15(16)$ & \\
\hline Delayed surgery & $3(3)$ & \\
\hline Late mortality & $7(7.5)^{*}$ & One related to pancreatitis \\
\hline Initial surgery & $12(13)$ & \\
\hline Initial surgical mortality & $1(1)$ & \\
\hline Initial mortality & 7 & \\
\hline Bleeding & 1 & \\
\hline Air embolism & 1 & \\
\hline Sepsis non-surgical & 4 & \\
\hline Postoperative (sepsis) & 1 & \\
\hline \multicolumn{3}{|l|}{ Late mortality } \\
\hline For unrelated reasons & 6 & \\
\hline Related to pancreas $\dagger$ & 1 & \\
\hline
\end{tabular}

*Six out of seven patients who died during follow-up were counted as having had initially successful pancreatic endotherapy (see table 2).

†Including endotherapy or surgery. 
centres. ${ }^{24}$ On the basis of the results of the present study, it therefore appears that endotherapy may confer a lower risk of complications. However, the variable severity of the condition may have automatically stratified severely ill patients to surgery and patients with less severe necroses to endotherapy. Although such selection bias is conceivable, it was also the case that patients who were deemed to be too ill for surgery were included in the series. To make valid conclusions about which patients should undergo which form of therapy, however, only a comparative and preferably randomised study would be able to answer this question. Due to the severity and complexity of both the condition and the interventions concerned, however, a randomised study of that type would have to be a multicentre one in order to recruit sufficient patient numbers and would probably be difficult to perform.

In any case, only prospective data using a uniform endotherapeutic treatment concept will reveal the effectiveness and complication rate of the endoscopic transluminal approach and clarify the differential indications between endotherapy and surgery. The data presented here are only preliminary, but suggest that with increasing standardisation and safety, transluminal endotherapy may become a less invasive and less risky alternative to primary surgical necrosectomy, with surgery being reserved for salvage treatment.

\section{Comment}

This manuscript originally described the long-term outcome of 115 patients from seven German centres. Twenty-two patients from one participating centre had to be withdrawn from this report after premature publication. ${ }^{25}$ Initial and long-term results from both cohorts (115 vs 93 patients) are without significant differences.

\section{Competing interests: None.}

Ethics approval: Ethics approval was given by the Ethik-Kommission der Länder Berlin-Brandenburg on 31 March 2008.

The GEPARD study is the German Multicenter Study on Endoscopic Pancreatic Retroperitoneal Debridement.

\section{REFERENCES}

1. de la Fuente SG, Demaria EJ, Reynolds JD, et al. New developments in surgery: natural orifice transluminal endoscopic surgery (NOTES). Arch Surg 2007;142:295-7

2. Seifert $\mathbf{H}$, Wehrmann T, Schmitt T, et al. Retroperitoneal endoscopic debridement for infected peripancreatic necrosis. Lancet 2000;356:653-5.
3. Kozarek RA, Brayko CM, Harlan J, et al. Endoscopic drainage of pancreatic pseudocysts. Gastrointest Endosc 1985;31:322-7.

4. Sahel J, Bastid C, Pellat B, et al. Endoscopic cystoduodenostomy of cysts of chronic calcifying pancreatitis: a report of 20 cases. Pancreas 1987:2:447-53.

5. Cremer $\mathbf{M}$, Devière J, Engelholm L. Endoscopic management of cysts and pseudocysts in chronic pancreatitis: long-term follow-up after 7 years of experience. Gastrointest Endosc 1989:35:1-9.

6. Binmoeller KF, Seifert $\mathbf{H}$, Walter A, et al. Transpapillary and transmural drainage of pancreatic pseudocysts. Gastrointest Endosc 1995:42:219-24.

7. Baron TH, Thaggard WG, Morgan DE, et al. Endoscopic therapy for organized pancreatic necrosis. Gastroenterology 1996;111:755-64.

8. Binmoeller KF, Soehendra N. Endoscopic ultrasonography in the diagnosis and treatment of pancreatic pseudocysts. Gastrointest Endosc Clin N Am 1995;5:805-16.

9. Giovannini M, Pesenti C, Rolland AL, et al. Endoscopic ultrasound-guided drainage of pancreatic pseudocysts or pancreatic abscesses using a therapeutic echo endoscope. Endoscopy 2001;33:473-7

10. Sanchez Cortes E, Maalak A, Le Moine 0, et al. Endoscopic cystenterostomy of nonbulging pancreatic fluid collections. Gastrointest Endosc 2002;56:380-6.

11. Kahaleh M, Shami VM, Conaway MR, et al. Endoscopic ultrasound drainage of pancreatic pseudocyst: a prospective comparison with conventional endoscopic drainage. Endoscopy 2006;38:355-9.

12. Baron TH. Endoscopic drainage of pancreatic fluid collections and pancreatic necrosis. Gastrointest Endosc Clin N Am 2003:13:743-64.

13. Charnley RM, Lochan $\mathrm{R}$, Gray $\mathrm{H}$, et al. Endoscopic necrosectomy as primary therapy in the management of infected pancreatic necrosis. Endoscopy 2006;38:925-8.

14. Raczynski S, Teich N, Borte G, et al. Percutaneous transgastric irrigation drainage in combination with endoscopic necrosectomy in necrotizing pancreatitis (with videos). Gastrointest Endosc 2006;64:420-4

15. Seewald S, Groth S, Omar S, et al. Aggressive endoscopic therapy for pancreatic necrosis and pancreatic abscess: a new safe and effective treatment algorithm (videos). Gastrointest Endosc 2005;62:92-100

16. Seifert H, Schuette A, Troschel P, et al. Transgastric endoscopic retroperitoneal debridement of peripancreatic necroses in 50 patients [abstract]. Gastrointest Endosc 2007;65:AB 206.

17. Papachristou GI, Takahashi N, Chahal $P$, et al. Peroral endoscopic drainage/ debridement of walled-off pancreatic necroses. Ann Surg 2007;245:943-51.

18. Traverso LW, Kozarek RA. Pancreatic necrosectomy: definitions and technique. J Gastrointest Surg 2005:9:436-9.

19. Uhl W, Warshaw A, Imrie C, et al. IAP Guidelines for the surgical management of acute pancreatitis. Pancreatology 2002;2:565-73.

20. Besselink MGH, Schoenmaeckers EJP, Buskens E, et al. Timing of surgical intervention in necrotizing pancreatitis: a 10 year consecutive case series and systematic review [abstract]. Pancreatology 2006:6:395-6.

21. Connor S, Alexakis N, Raraty MG, et al. Early and late complications after pancreatic necrosectomy. Surgery 2005;137:499-505.

22. Catto JW, Alexander DJ. Pancreatic debridement in a district general hospital viable or vulnerable? Ann R Coll Surg Engl 2002;84:309-13.

23. Nieuwenhuijs VB, Besselink MG, van Minnen LP, et al. Surgical management of acute necrotizing pancreatitis: a 13-year experience and a systematic review. Scand J Gastroenterol Supp/ 2003;239:111-6.

24. Slavin J, Ghaneh P, Sutton R, et al. Management of necrotizing pancreatitis. World J Gastroenterol 2001:7:476-81.

25. Hocke M, Will U, Gottschalk $P$, et al. Transgastral retroperitoneal endoscopy in septic patients with pancreatic necrosis or infected pancreatic pseudocysts. Z Gastroenterol 2008;46:1363-8. 